\title{
OPEN TENSION FREE MESH REPAIR OF INGUINAL HERNIAS: THE LICHTENSTEIN TECHNIQUE-OUR EXPERIENCE FOR LAST TEN YEARS
}

\author{
MA Rahman', MSH Sheikh¹, MI Siddique', KM Morshed', AKM Ahsan Ullah², Md. Shahidul Islam³
}

\begin{abstract}
Objective : Recurrences following hernia repair is one of the significant problem for both patient \& surgeon. Open tension free mesh techniques of inguinal hernia repair offers good results. Prosthetic materials have been increasingly used in hernia repair to prevent recurrences. Their use has been associated with several advantages, such as less postoperative pain, rapid recovery and low recurrence rates.

Materials and method : In this retrospective study, 1050 tension-free inguinal hernia repairs were performed between January 2001 and December 2010 in various medical institutes including BSMMU, DMCH \& some private clinics in Dhaka city, using a polypropylene mesh (Lichtenstein technique). The main outcome measure was early and late morbidity and especially recurrence.

Results : Inguinal hernia was indirect in $76 \%$ of cases (798 patients), direct in $22 \%$ (231 patients) and of the pantaloon (mixed) type in $2 \%$ (21 patients). Mean patient age was 44.5 years (range, $18-85)$. Follow-up was completed in 630 patients $(60 \%)$ by clinical examination or phone call. The median follow-up period was 2.5 years (range 1 - 5 years). Seroma and hematoma formation requiring drainage was observed in 25 and 8 patients respectively, while transient testicular swelling occurred in15 patients. Infection was found in 9 patients. Rejection of mesh occurred in 4 patients. There was 6 recurrence of the hernia (in one of these patients with late mesh rejection) (recurrence rate $=0.57 \%$ ). Postoperative neuralgia was observed in 25 patients $(2.38 \%)$.

Conclusion : Lichtenstein tension-free mesh repair of inguinal hernia is a simple, safe, comfortable, effective method.Tension free repair using synthetic meshes have been reported to result in better patient comfort and lower recurrence rates compared with the conventional procedures like Shouldice's or Bassini's operation ${ }^{2-4}$. Therefore it is the preferred method for hernia repair since 1994.
\end{abstract}

\section{Introduction}

Inguinal hernia repair is one of the most common surgical procedures that are used in the world ${ }^{1}$. The preferred surgical technique for inguinal hernia repair depends on the hand of the surgeon. In inguinal hernia repair, different laparoscopic and open techniques of tension free repair using synthetic meshes have been

1. Associate Professor, Dept. of Surgery, BSMMU, Dhaka

2. Assistant Professor, Dept. of Surgery, BSMMU, Dhaka

3. Medical Officer, Dept. of Surgery, BSMMU, Dhaka

Correspondence to: Dr. Md. Atiar Rahman Associate Professor of Surgery, Department of Surgery, Bangabandhu Sheihk Mujib Medical University, Shahbag, Dhaka

Received: 18 July 2012

Accepted: 01 October 2012 reported to result in better patient comfort and lower recurrence rates compared with the conventional procedures like Shouldice's or Bassini's operation ${ }^{2-4}$. Both laparoscopic and Lichtenstein open tensionfree (LO) hernia repairs have been shown to offer faster recovery and lower recurrence rates than the traditional methods. In an attempt to reduce the incidence of recurrences and to reinforce the plastic reconstruction various techniques have been used, including autologous tissue techniques and a vari ety of biomaterials ${ }^{5-6}$. Usher proposed the use of highdensity polyethylene to repair tissue defects of the 
chest and abdominal wall, about half a century ago ${ }^{7-}$ 8 . Since that time, a clear preference for synthetics has been observed and during the last decade a marked interest in the use of prosthetic materials was evident. The reports by Stoppa et $\mathrm{al}^{9}$ and by Lichtenstein ${ }^{10}$, as well as the innovation of laparoscopic hernia repair ${ }^{11-12}$, where the use of prostheses was associated with many advantages, greatly contributed to this change in our surgical philosophy. In this paper, we review our experience on tension - free surgical repair of a consecutive series of inguinal hernias using a polypropylene mesh (Lichtenstein technique).

\section{Method}

From January 2001 and December 2011, 1050 tension free repairs of inguinal hernia were performed by using a polypropylene mesh. Thirty patients had bilateral inguinal hernias. Inguinal hernia was indirect in $76 \%$ of cases (798 patients), direct in $22 \%$ (231 patients) and of the pantaloon (mixed) type in 2\% (21 patients). Mean patient age was 44.5 years (range, $18-85)$. All cases were performed under epidural or spinal anaesthesia. The exclusion criteria were presence of infection, the coagulation defects and age below 18 years. A single intravenous dose of $2 \mathrm{gm}$ of cephalosporine was administered to all the patients for infection prophylaxis preoperatively. Lichtenstein open hernia repair was performed using a technique that has been described in detail by Lichtenstein and Amid $^{13-15}$. Indirect hernia sacs were either dissected, ligated, cut at the neck, and then inverted or dissected and inverted with respect to the condition of the sac and the hernia orifice. The hernial sac was simply inverted in cases of direct hernias. A $7.515 \mathrm{~cm}$ polypropylene mesh (Prolene, Ethicon) was trimmed, sutured to the aponeuretic tissue over the pubic bone, to the shelving edge of the inguinal ligament, and to the internal oblique aponeurosis with 2-0 polypropylene (Prolene, Ethicon). A slit was made at the lateral end of the mesh for the spermatic cord. Operating time was measured from the start of the skin incision to the last skin suture. Follow-up examinations were performed after discharge on the 7th postoperative day, and after 1, 2, 6, 12, 18, 24 months, respectively. Further evaluation of all the patients for recurrences for 3 years were planned. Postoperative pain was measured by using Visual Analogue Scale (VAS) which scores the pain on a scale of 1 to 10 in which the score 1 represents no pain and the score 10 is the most intensive pain. The pains were marked by the patient pointing the score on the scale that best described his pain at that time. The pain scores using VAS at rest and in exercise which consisted of walking a definite distance, sitting in and standing up of a chair were recorded for each patient at predetermined time intervals of 6 and/or 12 , 24, 48 hours, 7 days, and 1 month after the operation. Analgesic medication was adjusted to the patients needs during the postoperative period. Tramadol $\mathrm{HCl}$ /ketorolac tromethamine were used intramuscularly (i.m.) as a postoperative analgesic for all patients. Oral Tramadol $\mathrm{HCl} / \mathrm{Ketorolac}$ tromethamine were recommended to the patients as postoperative analgesia after discharge when required. The number of the analgesic injections while in the hospital and days the patient needs as oral analgesic after discharge were recorded for each patient. The length of the hospital stay for each patient was also recorded. Data collection was carried out during pre and postoperative office visits. The patients were asked a series of questions with regard to experience of pain, the site or sites where pain was felt, analgesic needs, the length of limitation to routine daily activities, and work .The testes, nerve status, incision mark, and the presence of recurrent hernia were examined. The presence of early or late complications were noted (such as wound erythema, hematoma, wound infection, urinary retention, seroma, hydrocele, paresthesia and recurrence). Hernia recurrence was defined as a detectable bulge found in the operated groin by the patient himself or by the examining doctor with or without symptoms .

\section{Result}

Postoperative pain was minimal and easily controlled by the use of single analgesics (as previously reported). In the immediate postoperative period we had 48 complications (morbidity $=4.57 \%$ ); hematoma and seroma formation, requiring drainage, were observed in 8 and 25 patients, respectively. Testicular swelling occurred in 15 patients (1.42\%), all of which settled. It should be emphasized that we have not observed abscess formation or acute infection related to the presence of the foreign body (mesh). Follow-up was completed in 630 patients $(60 \%)$ by clinical examination $(n=602)$ or phone call $(n=28)$. The median follow-up period was 2.5 years (range $1-5$ years). Infection was found in 9 patients. In 4 patients we observed a delayed rejection of the mesh. This 
rare and interesting complication was presented by the late formation of a productive sinus at the site of the surgical incision. In all patients, a surgical debridement of this sinus tract was performed, but the fluid production continued. The mesh was then removed. The mesh was almost intact in all cases, without having caused the typical inflammatory response, resulting in mesh incorporation into the host tissues; as is well known, this is considered a significant advantage of the mesh repair over the traditional methods of hernia repair. There was 6 recurrence of the hernia (in one of these patients with late mesh rejection)(recurrence rate $=0.57 \%$ ). Severe postoperative neuralgia, persisting over 6 months postoperatively and requiring analgesics administration, was observed in 25 patients (2.38 \%). Management was conservative in all cases (by using simple, non-narcotic analgesics, such as NSAIDS) and progressively settled in all cases.

Table

(Complications)

\begin{tabular}{lc}
\hline Intraoperative Complications & $\mathrm{LO}(\mathrm{n}=1050)$ \\
Bleeding & 20 \\
Divided Inguinal Nerve & 9 \\
TOTAL & 29 \\
Postoperative Complications & $\mathrm{LO}(\mathrm{n}=1050)$ \\
Urinary Retention & 4 \\
Hematoma & 8 \\
Seroma & 25 \\
Hydrocele & 15 \\
Wound Infection & 9 \\
Persistent Pain/Tenderness & 25 \\
Paresthesia & 13 \\
\hline Total & 99 \\
\hline
\end{tabular}

\section{Discussion}

The description of the Lichtenstein tension -free mesh repair, about 19 years ago, opened a new era in groin hernia repair ${ }^{10}$. Postoperative pain is minimal, as a result of the tension-free technique. The method is very simple, effective, is associated with a very low recurrence rates (ranging from 0 to $2 \%$ in the literature) and can be performed under local or regional anaesthesia ${ }^{16-18}$. For these important advantages, it is currently the preferred method for the plastic reconstruction of inguinal hernias for the majority of surgeons around the world.
A variety of prosthetic mesh is available in the market. The ideal mesh properties are inertness, resistance to infection, molecular permeability, pliability, transparency, mechanical integrity, and biocompatibility. Absorbable mesh does not remain in the wound long enough for adequate collagen to be deposited, while multi-filament mesh can harbour bacteria. Monofilament mesh is the most popular presently in use with the various types of polypropylene having different characteristic advantages ${ }^{18}$. Use of porous mesh (polypropylene) allows a large surface area for in-growth of connective tissue leading to permanent fixation of the prosthesis within the abdominal wall. Intra parietal placement of the prosthesis allows well vascularized, tissue coverage of all aspects of the prosthesis. Fears of complications related to mesh implantation have proved to be without foundation. The use of vacuum drains is indicated in large inguinal hernias in order to minimize hematoma or seroma formation. However, duration of antibiotic use or indication for suction drainage differ among investigators. To reduce the chance of recurrence, the mesh should extent 2-4 $\mathrm{cm}$ beyond the boundary of Hesselbach's triangle ${ }^{17}$. The position of the mesh beneath the aponeurosis of the external oblique results in the intra abdominal pressure working in favour of the repair, since the external oblique aponeurosis keeps the mesh tightly in place by acting as an external support when intraabdominal pressure rises. The mesh should be fixated carefully, by the use of Prolene sutures or staples, to prevent folding, wrinkling, or curling of the mesh around the cord.

\section{Conclusion}

The method is simple, can be performed by all the surgeons - even those without special interest in hernia surgery and is very effective in the prevention of recurrences. Indeed, an extremely low recurrence rate (range, $0-0.7 \%$ ) has been reported from many groups of surgeons ${ }^{16,19-21}$. The method combines many advantages, such as simplicity, effectiveness, safety, comfortable postoperative course with easily controlled pain, rapid return to unrestricted activities, an impressively low recurrence rate and high patient satisfaction. We have been encouraged by these good results of this procedure in a relatively large number of patients $(n=1050)$. For these reasons, it is our preferred method for hernia repair since 1994. 


\section{References}

1. Heikkinen T.J., Haukipuro K., Koivukangas P., Hulkko A. A Prospective Randomized Outcome and Cost Comparison of Totally Extra peritoneal Endoscopic Hernioplasty Versus Lichtenstein Hernia Operation Among Employed Patients. Surg Laparosc Endosc, 1998; 8 : 338-44.

2. Shulman A. G., Amid P. K., Lichtenstein I. L. The safety of mesh repair for primary inguinal hernias : results of 3,019 operations from five diverse surgical sources. Am Surg, 1992; 58 : 255-7.

3. Wantz G. E. Experience with the tension-free hernioplasty for primary inguinal hernias in men. J Am Coll Surg, 1996; 183 : 351-6.

4. Memon M. A., Rice D., Donahue J. H. Laparoscopic herniorraphy. J Am Coll Surg, 1997; 184 : 325-35.

5. Bassini E. Sulla cura radicale dell'ernia injuinale. Arch Soc Ital Chir. 1887; 4: 380-388.

6. DeBord JR. The Historical development of prosthetics in hernia surgery. Surg Clin North Am. 1998; 78: 973-1006.

7. Usher FC. A new plastic prosthesis for repairing tissue defects of the chest and abdominal wall. Am J Surg. 1959; 97: 629-635.

8. Usher FC, Fries JC, Ochsner JL, Tuttle LLD., Jr Marlex mesh a new plastic mesh for replacing tissue defects II. Arch Surg. 1959; 78: 138-145

9. Stoppa RE, Petit J, Henry X. Unsutured Dacron prosthesis in groin hernias. Int Surg.1975;60:411419.

10. Lichtenstein IL, Shulman AG, Amid PK, et al. The tension free hernioplasty. Am J Surg. 1989; 157: 188-193.
11. Popp LW. Endoscopic patch repair of inguinal hernia in a female patient. Surg Endosc. 1990; 4: 10-12.

12. Ramshaw BJ, Tucker JG, Duncan TD. Laparoscopic herniorrhaphy: A review of 900 cases. Surg Endosc. 1996;10:255-232. doi: $10.1007 /$ s 004649910006.

13. Lichtenstein I. L. Herniorraphy A Personal Experience With 6,321 Cases. Am J Surg, 1987, $153: 553-9$

14. Lichtenstein I. L., Shulman A. G., Amid P. K., Montllor M. M. The Tension-Free Hernioplasty. Am J Surg, 1989; 157 : 188-93.

15. Amid P. K., Shulman A. G., Lichtenstein I. L. Critical Scrutiny of the Open "Tension-Free" Hernioplasty. Am J Surg, 1993, 165 : 369-71.

16. Kurzer M, Belsham PA, Kark AE. The Lichtenstein repair. Surg Clin North Am. 1998; 78: 1025-1046.

17. Amid PK, Shulman AG, Lichtenstein IL. Open "Tension-Free" repair of inguinal hernias; The Lichtenstein technique. Eur J Surg. 1996; 162: 447-453.

18. Goldstein HS. Selecting the right mesh. Hernia. 1999; 3: 23-26.

19. Amid PK, Shulman AG, Lichtenstein IL. Simultaneous repair of bilateral inguinal hernias under local anesthesia. Ann Surg. 1996; 223: 249-252.

20. Capozzi JA, Berkenfield JA, Cheaty JK. Repair of inguinal hernia in the adult with prolene mesh. Surg Gynecol Obstet. 1988;167:124-128.

21. Shulman AG, Amid PK, Lichtenstein IL. A survey of non-expert surgeons using the open tensionfree mesh repair for primary inguinal hernias. Int Surg. 1995; 80:35-36 